\title{
Development of a monoenergetic ultraslow antiproton beam source for high-precision investigation
}

\author{
N. Kuroda, ${ }^{1}$ H. A. Torii, ${ }^{1}$ Y. Nagata, ${ }^{2}$ M. Shibata, ${ }^{3}$ Y. Enomoto, ${ }^{2}$ H. Imao, ${ }^{4}$ Y. Kanai, ${ }^{2}$ M. Hori, ${ }^{5}$ H. Saitoh, ${ }^{6}$ \\ H. Higaki, ${ }^{7}$ A. Mohri, ${ }^{2}$ K. Fujii, ${ }^{1}$ C. H. Kim, ${ }^{1}$ Y. Matsuda,${ }^{1}$ K. Michishio,${ }^{8}$ Y. Nagashima,${ }^{8}$ M. Ohtsuka, ${ }^{1}$ \\ $\mathrm{K}$. Tanaka, ${ }^{1}$ and Y. Yamazaki ${ }^{1,2}$ \\ ${ }^{1}$ Graduate School of Arts and Sciences, University of Tokyo, Meguro, Tokyo 153-8902, Japan \\ ${ }^{2}$ Atomic Physics Laboratory, RIKEN, Wako, Saitama 351-0198, Japan \\ ${ }^{3}$ Institute of Particle and Nuclear Studies, KEK, Tsukuba, Ibaraki 305-0801, Japan \\ ${ }^{4}$ Nishina Center for Accelerator-Based Science, RIKEN, Wako, Saitama 351-0198, Japan \\ ${ }^{5}$ Max-Planck-Institut für Quantenoptik, D-85748, Garching, Germany \\ ${ }^{6}$ Department of Advanced Energy, University of Tokyo, Kashiwa, Chiba 277-8561, Japan \\ ${ }^{7}$ Graduate School of Advanced Sciences of Matter, Hiroshima University, Higashi-Hiroshima, Hiroshima 739-8530, Japan \\ ${ }^{8}$ Department of Physics, Tokyo University of Science, Shinjuku, Tokyo 168-8601, Japan
}

(Received 13 July 2011; published 17 February 2012)

\begin{abstract}
The ASACUSA collaboration developed an ultraslow antiproton beam source, monoenergetic ultraslow antiproton source for high-precision investigation (MUSASHI), consisting of an electromagnetic trap with a liquid He free superconducting solenoid and a low energy antiproton beam transport line. The MUSASHI was capable of trapping and cooling more than $1 \times 10^{7}$ antiprotons and extracting them as an ultraslow antiproton beam with energy of $150-250 \mathrm{eV}$.
\end{abstract}

DOI: 10.1103/PhysRevSTAB.15.024702

PACS numbers: 37.10.Ty, 07.77.Ka, 52.27.Jt

\section{INTRODUCTION}

An extremely low energy antiproton $(\bar{p})$ beam source is an important tool to synthesize antihydrogen $(\overline{\mathrm{H}})$ atoms [1-3] and antiprotonic atoms $\left(\bar{p} \mathrm{~A}^{+}\right)$. The studies of these exotic atoms can test $C P T$ invariance and the weak equivalence principle [4]. The collision dynamics by such low energy $\bar{p}$ can reveal such exotic antiprotonic atom formation [5] and the ionization processes of atoms and molecules [6,7]. The study of the nuclear surface via $\bar{p} \mathrm{~A}^{+}$ formation and annihilation is also considered $[8,9]$. On the other hand, such exotic atoms can only be efficiently synthesized from component particles at $\mathrm{eV}$ and lower energy scales, and this is far below the mandatory $\mathrm{GeV}$ scale of accelerator-produced $\bar{p}$.

This energy gap has been partially bridged by the CERN Antiproton Decelerator (AD), which decelerates $\bar{p}$ s with $\mathrm{GeV}$-scale kinetic energy to $5.3 \mathrm{MeV}$, and re-ejects them every $110 \mathrm{~s}$ in $150 \mathrm{~ns}$ long pulses, containing $3 \times 10^{7}$ particles. This beam energy is still far above the chemical energy scale. One solution is the use of so-called trap-based beams, as applied to the case of $e^{+}[10]$ or radioactive nuclei (RI) [11]. The ASACUSA collaboration has proposed [12] and developed a trap-based ultraslow $\bar{p}$ beam source $[13,14]$ for these experiments.

Published by the American Physical Society under the terms of the Creative Commons Attribution 3.0 License. Further distribution of this work must maintain attribution to the author(s) and the published article's title, journal citation, and DOI.

\section{APPARATUS}

\section{A. Overview}

Conventional $\bar{p}$ catching traps working at the $\mathrm{AD}$ especially designed for production of $\overline{\mathrm{H}}$ atoms use approximately $70 \mathrm{mg} / \mathrm{cm}^{2}$ metal foils to further reduce the $\bar{p}$ energy from the $\mathrm{MeV}$ scale to the $\mathrm{keV}$ scale [15-18]. However, many incident $\bar{p}$ typically $99 \%$ stop and annihilate within such foils, while others have too high an energy to be captured in the potential well of the trap located downstream. Instead of these simple degrader foils, we used a radio frequency quadrupole decelerator (RFQD) which was connected to the monoenergetic ultraslow antiproton source for highprecision investigation (MUSASHI) with a low energy beam transport line (LEBT), as schematically shown in Fig. 1.

The RFQD, $3.5 \mathrm{~m}$ in length, reduced the $5.3 \mathrm{MeV} \mathrm{AD}$ beam energy to $10-120 \mathrm{keV}$ by biasing its deceleration cavity at a voltage of $\left|V_{\mathrm{RFQD}}\right| \lesssim 60 \mathrm{kV}$. Some of the antiprotons, typically $30 \%$ for each $\mathrm{AD}$ shot, fulfilled the phase condition necessary for deceleration by the RFQD, the remainder being transmitted without deceleration [19]. The $\bar{p}$ beam passing through the LEBT was steered and focused by a set of dipoles and solenoids. It was observed that the reduced $\bar{p}$ energy allowed a much larger number of emerging $\bar{p}$ to be captured in the MUSASHI placed after the RFQD than is normally the case for simple energy degrader foils.

Figure 2 shows the MUSASHI based on a multiring electrode trap (MRT) [20] which is composed of two parts, a stack of multiple ring electrodes (MRE) housed in an ultrahigh vacuum (UHV) bore and a superconducting 


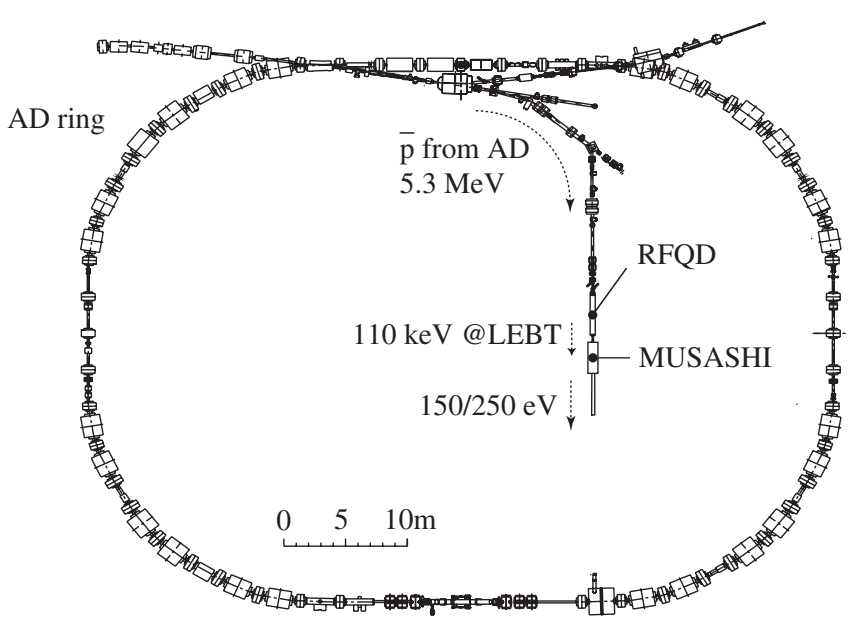

FIG. 1. Scheme of the MUSASHI sequentially combined with the AD, the RFQD, and the LEBT. The AD provides $5.3 \mathrm{MeV}$ antiprotons. The RFQD decelerates them to $110 \mathrm{keV}$ and transports them to the MUSASHI via the LEBT. The antiprotons are trapped and cooled in the MUSASHI, and then extracted at 150 or $250 \mathrm{eV}$.

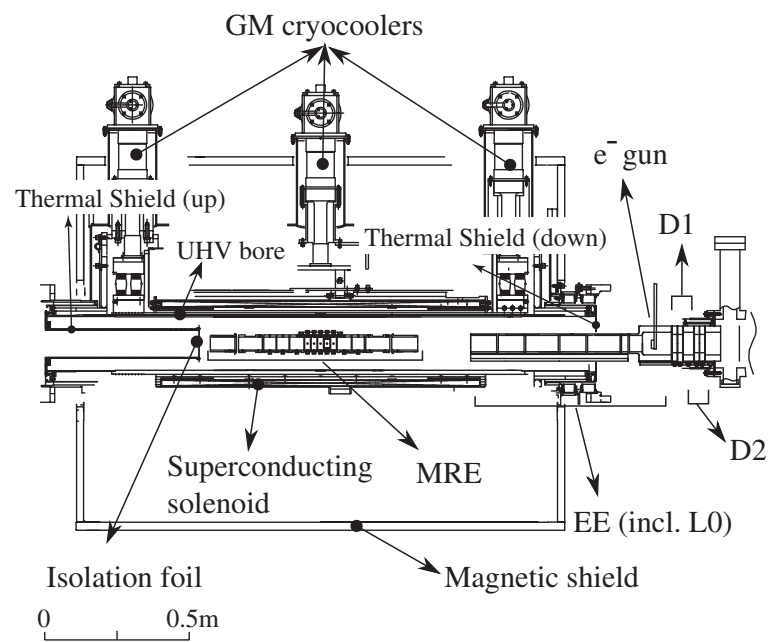

FIG. 2. Cross sectional view of MUSASHI and the ultraslow beam transport line.

solenoid. The $\bar{p}$ s were captured and cooled inside the MRE. Then the cold cloud of $\bar{p}$ was extracted via extraction electrodes (EE) in order to be used in the abovementioned various experiments.

The MUSASHI was designed to work in two operation modes for $\bar{p}$ extraction. Long bunched $\bar{p}$ beams for atomic collision experiments [5-7] were obtained by the DC extraction mode. Pulsed beams from the MUSASHI were obtained by the pulse extraction mode, which was used for $\overline{\mathrm{H}}$ experiments [3].

\section{B. Superconducting solenoid with a UHV bore and an isolation foil}

The MUSASHI superconducting magnet yields a magnetic field as high as $5 \mathrm{~T}$ with a uniformity of $0.1 \%$ over
$635 \mathrm{~mm}$ (length) $\times 10 \mathrm{~mm}$ (diameter). The MUSASHI superconducting magnet was cooled by a thermally connected 1 W Gifford-McMahon (GM)-type cryocooler (SRDK-408D2) via oxygen-free high thermal conductivity (OFHC) copper bar, so that MUSASHI is operating without any filling of liquid helium. The magnetic field strength was chosen to optimize the combined efficiency of $\bar{p}$ trapping and extraction. Since the experimental apparatus for $\overline{\mathrm{H}}$ formation operated at $2.5 \mathrm{~T}$ and $\bar{p}$ almost follows the magnetic field line adiabatically, the field strength was chosen to $2.5 \mathrm{~T}$. The MUSASHI solenoid was supported at both ends where its position can be adjusted vertically and horizontally, which enabled alignment of the solenoid within $\pm 2 \mathrm{~mm}$ against the UHV bore axis for stable confinement and efficient $\bar{p}$ extraction.

The UHV bore was $1725 \mathrm{~mm}$ long and had a diameter of $164.7 \mathrm{~mm}$, and was made by nonmagnetic stainless steel clad in $\mathrm{Cu}$ strips. The temperature of the UHV bore was homogeneously kept at around $4 \mathrm{~K}$ by two $1.5 \mathrm{~W}$ GM-type cryocoolers (SRDK-415D) which were thermally connected to the bore through a OFHC $\mathrm{Cu}$ mesh. At both ends of the UHV bore as shown in Fig. 2, two thermal shields connected to $50 \mathrm{~K}$ stages were installed in order to reduce thermal radiation from outside at room temperature. The temperature difference between the center and the ends of the UHV bore where GM cryocoolers were connected was $0.2 \mathrm{~K}$. Such a cold environment was one of the important conditions for stable confinement of $\bar{p}$ to avoid losses caused by annihilations with any residual gases.

The vacuum conditions in the RFQD through which the $\bar{p}$ passed only once was around $10^{-7} \mathrm{~Pa}$. Two $90 \mu \mathrm{g} / \mathrm{cm}^{2}$ biaxially oriented polyethylene terephtalate (Bo-PET) foils located $37.4 \mathrm{~cm}$ upstream of the MRE center were therefore used to isolate the trap vacuum at $\leq 10^{-10} \mathrm{~Pa}$ from the RFQD vacuum.

For diagnosis purposes, ten thin $\mathrm{Ag}$ strips of $25 \mathrm{~nm}$ thickness and $940 \mu \mathrm{m}$ width were printed onto each BoPET foil as shown in Fig. 3 [21]. The strips were set perpendicular to that of the other foil. Beam profiles were measured by reading charges induced by the antiprotons on each strip with amplifiers [22]. The steered and focused beam was diagnosed by this "foil detector."

\section{Multiring electrode trap}

As shown in Fig. 4, the MRE is comprised of 14 ring electrodes with radius of $R=20 \mathrm{~mm}$ aligned on the axis of a superconducting solenoid. The MRE can generate almost any axisymmetric potential where even a selfcharge potential of a plasma can be compensated [20]. This feature allows stable confinement of a large number of charged particles [23]. For confinement of charged particles, five center electrodes, HE1-5, and one neighboring electrode on each side, UE3 and DE1, were used. By applying proper voltages on each electrode as shown in Fig. 4, an electrostatic harmonic potential was produced 


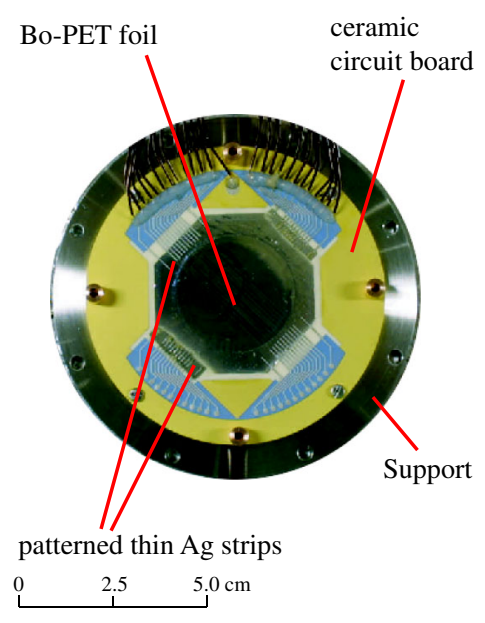

FIG. 3. Foil detector.

with the axial length of $2 L=125 \mathrm{~mm}$, as described in the cylindrical coordinate $(\rho, z)$ [24],

$$
\phi(\rho, z)=-V_{0} \frac{\rho^{2}-2 z^{2}}{2 L^{2}+R^{2}}+\delta,
$$

where the potential difference was $V_{0}=\phi(0, L)-\phi(0,0)$, and the offset from the surface potential on the center electrode was $\delta$. Figure 5(a) shows the resultant potential along the axis for $V_{0}=10 \mathrm{~V}$. A quadratic function is superimposed on the produced harmonic potential. The difference between the produced potential along the axis and the quadratic function is shown in Fig. 5(e). The harmonic potential region by the MRE is much longer than in conventional Penning traps. The difference for $\rho=0 \mathrm{~mm}$ is within $0.3 \%$ for the center $7.6 \mathrm{~cm}$ region. The calculated potentials and their differences at $\rho=5,10$, and $15 \mathrm{~mm}$ are also shown in Figs. 5(b)-5(d) and 5(f)-5(h), respectively. As is expected, the produced potential distorted at the larger $\rho$. For the case of $\rho=5 \mathrm{~mm}$, the distortion is still within $0.4 \%$. However, for $\rho=10$ or $15 \mathrm{~mm}$, the difference amounts to $2 \%-7 \%$. The voltage, $V_{0}$, was chosen to $50 \mathrm{~V}$ during the following experiments.

The DC outputs from signal generators (NF 1946) were amplified by high slew rate power amplifiers (APEX PA84) and applied on the ring electrodes. These outputs were interfaced via general purpose interface bus to a control system. Fine stainless steel (SUS 316L) wires of $0.2 \mathrm{~mm}$ diameter were selected as signal cables in order to reduce heat conduction. The wires were shielded by annealed stainless steel (SUS 304) tube and insulated with polyimide tubes of $0.04 \mathrm{~mm}$ thickness, which worked as coaxial cables to reduce cross talk.

Before the injection of a $\bar{p}$ beam, electrons as coolant were injected from the downstream side of the MRE. They were emitted from a barium dispenser cathode mounted on a pneumatic-type linear feedthrough as shown in Fig. 2, which was retracted during the extraction procedure of ultraslow $\bar{p}$ beams. The typical number of stored $e^{-}$was $4 \times 10^{8}$. The cloud of $e^{-}$forms a non-neutral plasma under a rigid rotor equilibrium in the harmonic potential well of the MRT.

The radial distribution of the $e^{-}$plasma was controlled by applying a rotating wall technique. One of the ring electrodes, HE4, was azimuthally segmented into 4 sectors to which radio frequency fields were applied with phases shifted by $\pi / 2 \mathrm{rad}$ from one segment to another, thereby generating a rotating electric potential wall as shown in Fig. 6. This rotating wall can exchange angular momentum with the plasma and thus radially compress or expand it [25]. For better cooling of the incident $\bar{p}$ beam, the radial distribution of the $e^{-}$plasma was adjusted to the size of the $\bar{p}$ beam, typically, $3.4 \mathrm{~mm}$ in radius and $1 \times 10^{8} \mathrm{~cm}^{-3}$ in density.

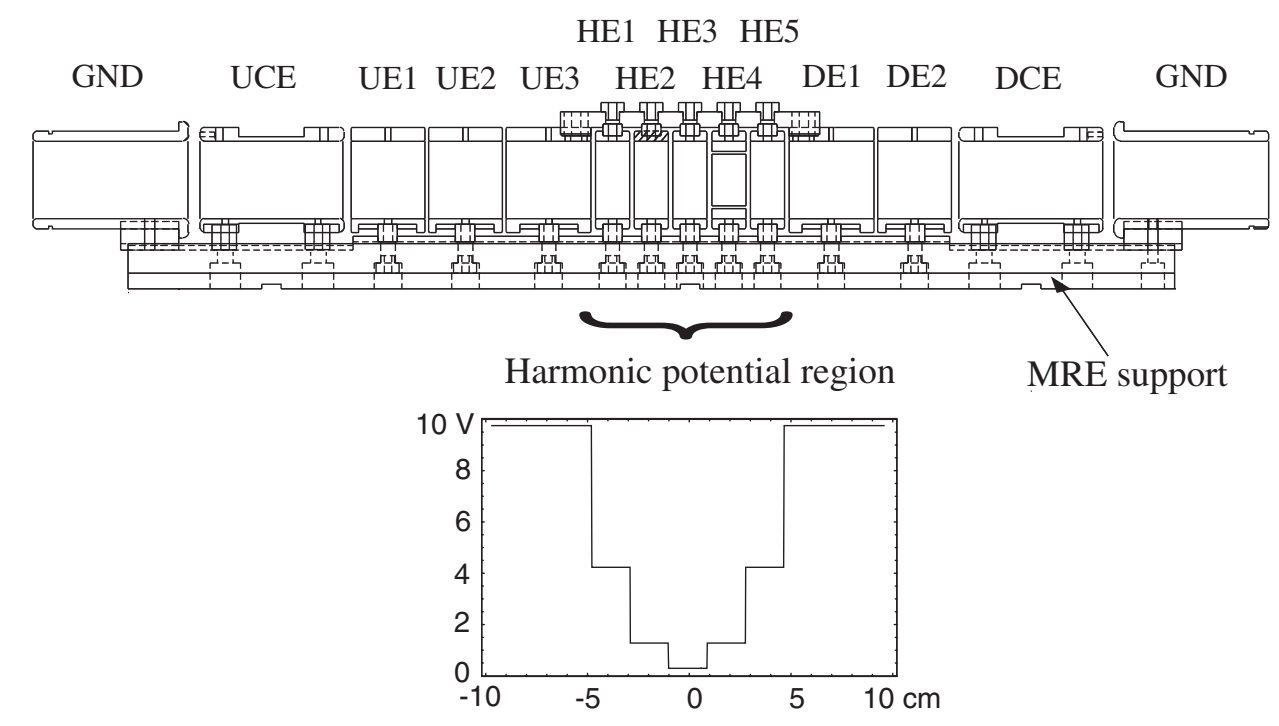

FIG. 4. Configuration of the MRE. The lower part shows the voltages applied on each ring electrode for a harmonic potential well. 


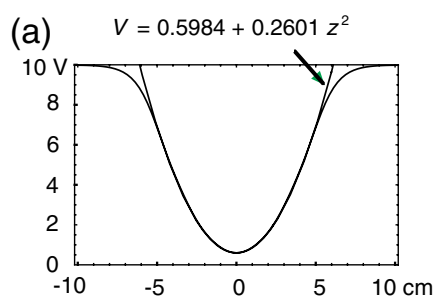

(e)

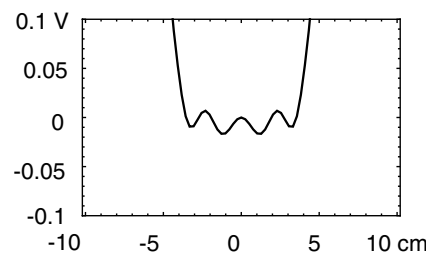

(b)

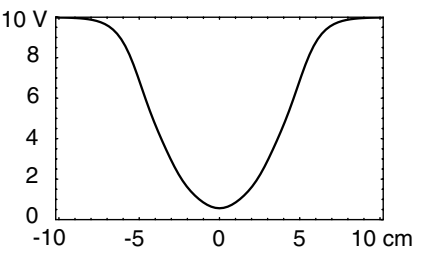

(f)

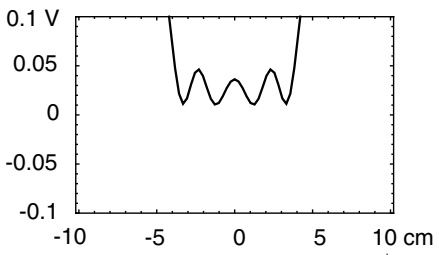

(c)

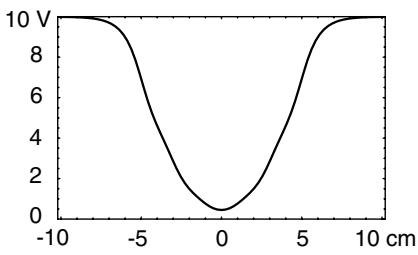

(g)

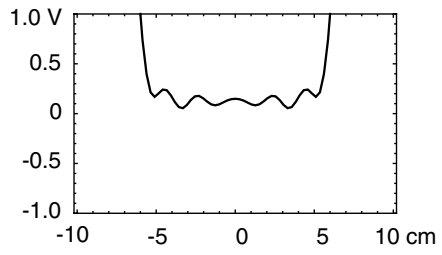

(d)

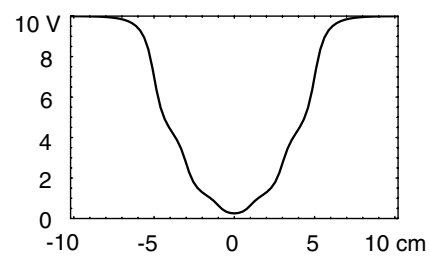

(h)

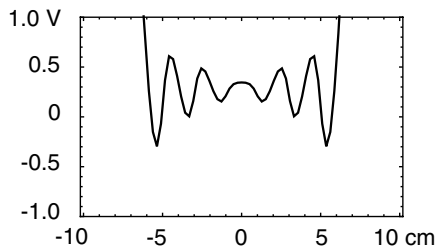

FIG. 5. (a) The potential produced at $\rho=0 \mathrm{~mm}$. A fitting quadratic function is also shown. (e) The difference between the produced potential and the quadratic function in the case of (a). (b) and (f) Potential and difference at $\rho=5 \mathrm{~mm},(\mathrm{c})$ and (g) at $\rho=10 \mathrm{~mm}$, and (d) and (h) at $\rho=15 \mathrm{~mm}$.

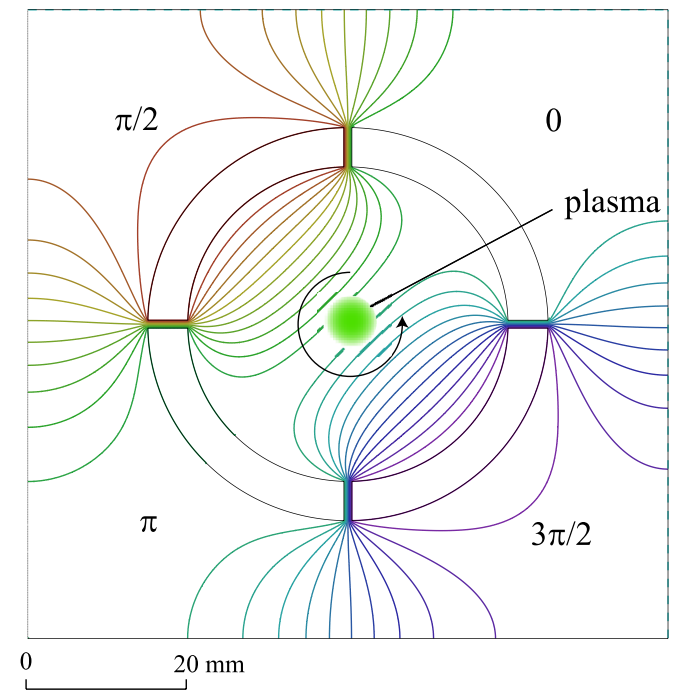

FIG. 6. Cross sectional view of the segmented electrode HE4 for applying a rotating wall. Equipotential lines are also shown.

In the case where the boundary of the spheroidal plasma at a finite temperature is still regarded as sharp, the dispersion relation of axially symmetric electrostatic oscillation gives relations between the aspect ratio, temperature, and density of the plasma $[13,26]$. Observed frequencies of electrostatic oscillations for the prepared plasma having the aspect ratio of 11 are shown in open circles in Fig. 7. The calculated frequencies were also shown. The good agreement between each data confirms that the shape of the plasma is nearly spheroid.

Figure 8 shows the experimental procedure of $\bar{p}$ capture, cooling, manipulation, and extraction from the MRE: First, as shown in Fig. 8(a), a potential, $-13 \mathrm{kV}$, was applied to the electrode downstream catching electrode (DCE), in

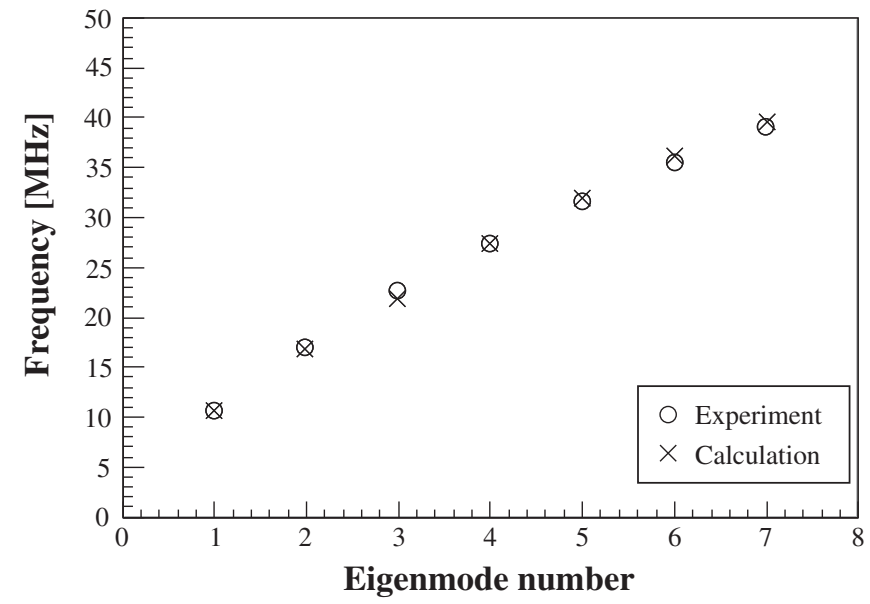

FIG. 7. Observed and calculated axially symmetric frequencies up to 7 th eigenmode.

order to reflect the pulsed $\bar{p}$ beam, where the distance between upstream catching electrode (UCE) and DCE was $318 \mathrm{~mm}$. The potential of UCE was applied after the passage of the $\bar{p}$ pulse with the rise time $\tau=300$ ns by a metal-oxide-semiconductor field-effect transistor switch to readily close the entrance by the time the pulse had bounced back. Then as shown in Fig. 8(b), collisions between the $\bar{p}$ s and the $e^{-}$plasma cooled the incident beam. Electrons soon lost their energy by cyclotron radiation because of strong magnetic field. Subsequently, as shown in Fig. 8(c) the trapped $e^{-} \mathrm{s}$ were removed during a time when the trapping potential was turned off, which was short enough to keep the $\bar{p}$ cloud in the trapping region but too long for $e^{-} \mathrm{s}$ to stay there. In the MUSASHI case, it was important to remove $e^{-}$from the trapping region for better $\bar{p}$ cloud extraction efficiency [13]. As shown in Fig. 8(d), the segmented electrode was also used to 
(a) trapping

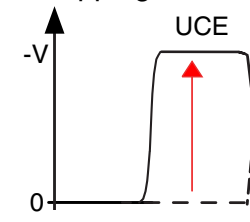

(b) cooling

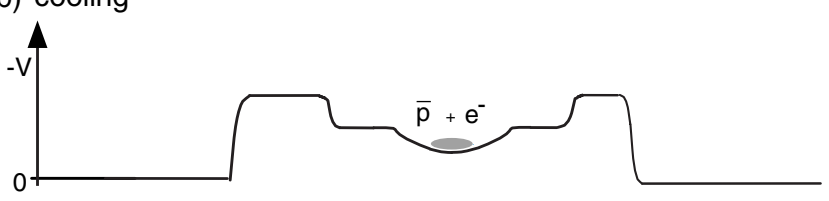

(c) $e^{-}$kickout

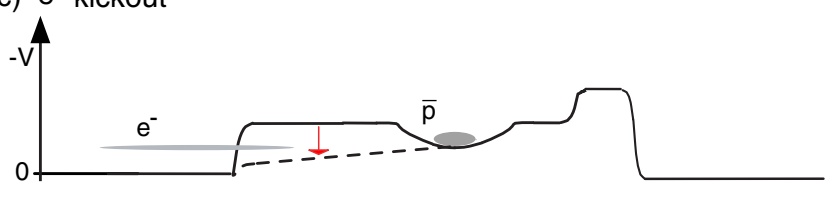

(d) compression

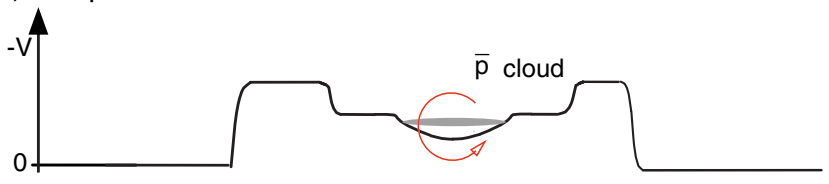

(e) extraction

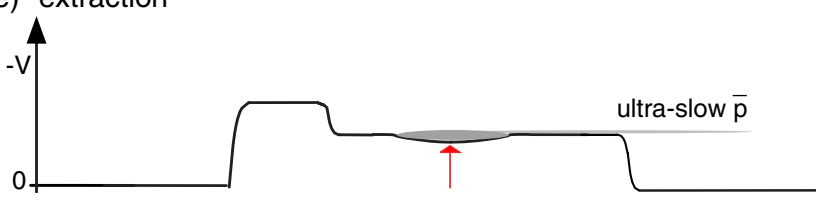

FIG. 8. Antiproton trapping, cooling, electron kick-out, compression, and extraction procedures.

compress the $\bar{p}$ cloud for better focusing [14]. In the case of DC extraction, as shown in Fig. 8(e), the trapping potential well was gradually ramped up while the barrier potential remained the same. The potential

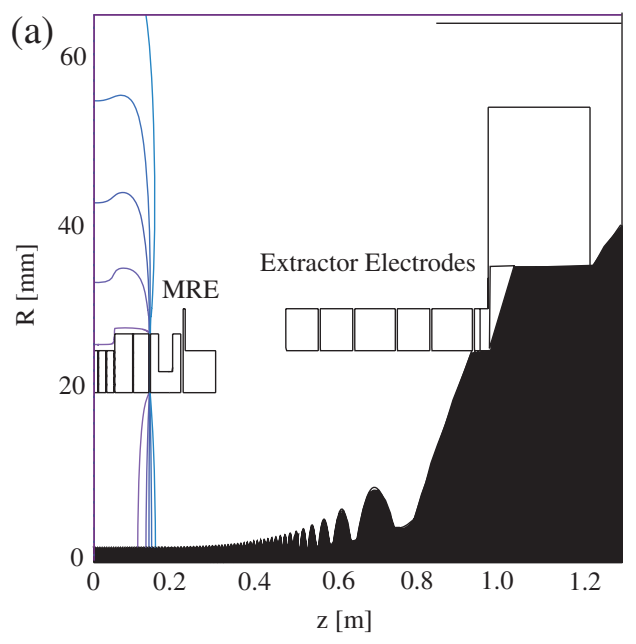

FIG. 9. Calculated envelopes of extracted $250 \mathrm{eV} \bar{p}$ beams $V_{\mathrm{L} 0}=905 \mathrm{~V}$. difference between the barrier potential and the ground defined the kinetic energy of extracted $\bar{p}$ beams. In the case of pulsed extraction, the cloud was extracted as a pulse by a quick ramp-up of the trapping potential.

\section{Extraction electrodes}

Extraction electrodes EE shown in Fig. 2 were installed in order to extract the cold $\bar{p}$ cloud as a slow beam from the UHV bore. After leaving the MRE, the $\bar{p}$ traveled with the same energy along the homogeneous magnetic field lines, and then entered the EE, where they were accelerated or decelerated. The extraction energy of the $\bar{p}$ beam was chosen as $250 \mathrm{eV}$ during the following experiments unless otherwise specified. When $\bar{p}$ s are extracted from the strong magnetic field, the trajectories tend to follow the magnetic field lines, which are strongly diverging. At the same time, the angular momenta of the extracted particles are increased when they leave the magnetic field. Since the magnetic components of the canonical angular momentum become larger than the thermal component after the $e^{-}$ cooling in MUSASHI [27], the key parameter to govern the emittance of the $\bar{p}$ beam is not the temperature of the cloud but the radius and the field strength. Three electrodes of the EE formed an electrostatic lens, L0, which compensates for the diverging trajectories. Figures 9(a) and 9(b) show calculated envelopes of extracted $250 \mathrm{eV} \bar{p}$ beam with its radius of $1.5 \mathrm{~mm}$ and transverse kinetic energy of $1 \mathrm{eV}$. When $L 0=0 \mathrm{~V}$ as shown in Fig. 9(a), a part of the $\bar{p}$ s hit electrodes of the EE. However, by applying a voltage, $905 \mathrm{~V}$, on L0, as shown in Fig. 9(b), all $\bar{p}$ s passed through the EE. A set of deflectors, D1 and D2 in Fig. 2, were installed for steering of the extracted beam.

\section{E. Transport beam line}

In order to transport the $\bar{p}$ beam, two types of beam line were developed. One was an electrostatic beam line which

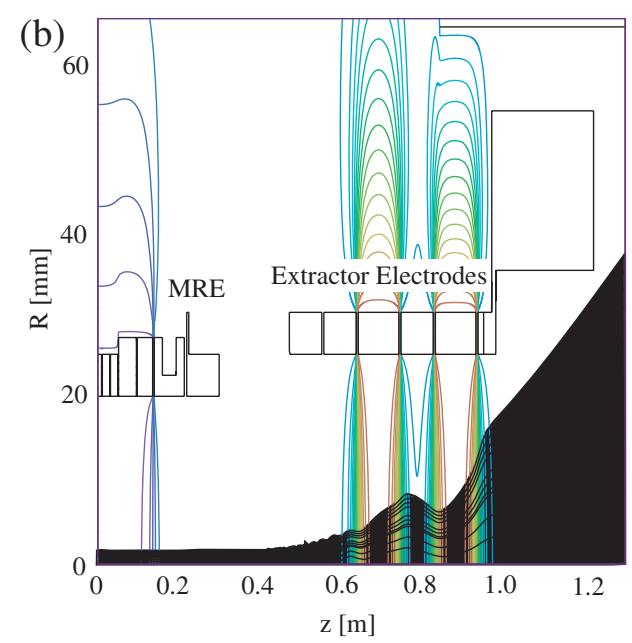

(a) without focusing by electrostatic lens, $V_{\mathrm{L} 0}=0$. (b) With 


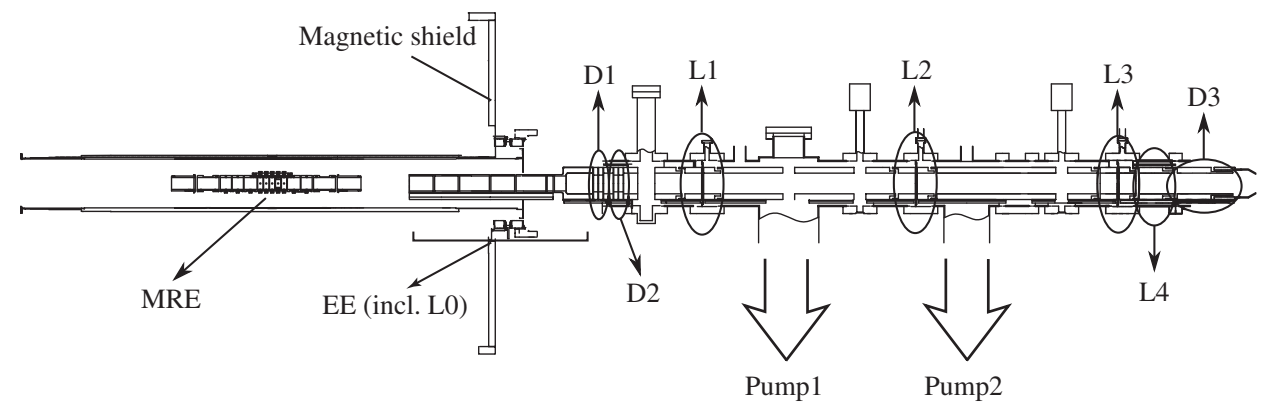

FIG. 10. Cross sectional view of the electrostatic transport beam line with MUSASHI.

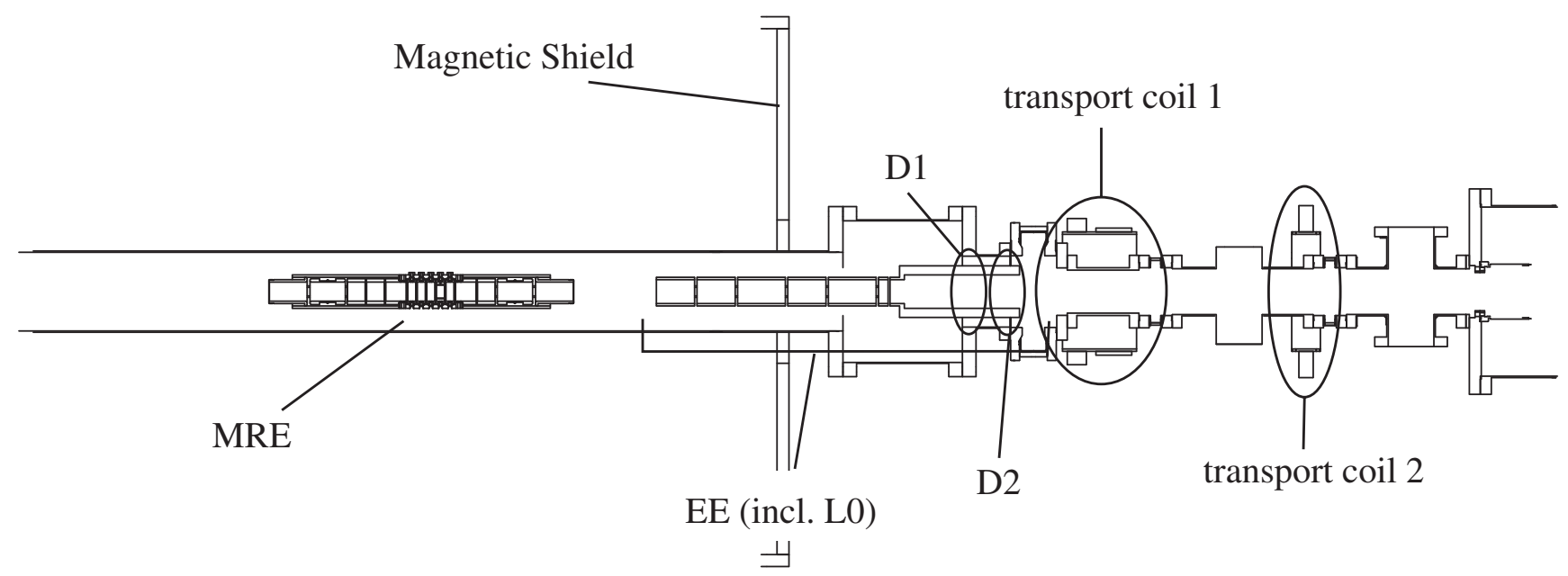

FIG. 11. Cross sectional view of the magnetostatic transport beam line with MUSASHI.

transported $\bar{p}$ s to experimental chambers using gaseous targets [27]. Another was a magnetostatic beam line for UHV chambers like the cusp trap [3] for $\overline{\mathrm{H}}$ formation.

As shown in Fig. 10, the electrostatic beam line was equipped with a three-stage differential pumping system in order to maintain a pressure lower than $10^{-10} \mathrm{~Pa}$ in the UHV bore while allowing a pressure of around $10^{-4} \mathrm{~Pa}$ in the chambers used for atomic collision experiments with gaseous targets [5-7]. The differential pumping system was realized by variable metallic apertures, which were designed to be electrically floatable in order to compose three sets of electrostatic lenses, L1-L3, for transportation and the last lens, L4, for focusing of $\bar{p}$ beams. The pumping systems were connected at the two locations, PUMP1 and PUMP2, indicated in Fig. 10. The first stage was evacuated from the PUMP1 by a tandem of $1500 \mathrm{l} / \mathrm{s}$ and $70 \mathrm{l} / \mathrm{s}$ turbo molecular pumps (TMPs), and the vacuum was kept at around $1 \times 10^{-7} \mathrm{~Pa}$ during the collision experiments. The second stage was evacuated from the PUMP2 by $400 \mathrm{l} / \mathrm{s}$ TMP and reached the order of $10^{-6} \mathrm{~Pa}$. For fine steering of transported beams, another deflector, D3, located at the end of the beam line was also used.

Figure 11 shows a schematic drawing of the magnetostatic beam line having a pair of transport coils which generated a magnetic field of up to $0.035 \mathrm{~T}$. The transport section was kept at the pressure of $10^{-7} \mathrm{~Pa}$.

\section{F. Detectors}

For diagnostic purposes it was important to know the position and time distribution of $\bar{p}$ annihilations during the capture, cooling, manipulation, extraction, and transportation. Two sets of track detectors consisting of $2 \mathrm{~m}$-long plastic scintillator bars were used for this purpose. They were placed parallel to the axis along the MRT at distances of 80 and $153.7 \mathrm{~cm}$ from the axis. They had cross sections of $4(\mathrm{H}) \times 6(\mathrm{~V}) \mathrm{cm}^{2}$. Coincident light pulses produced in these scintillators by the passage of charged particles from nearby $\bar{p}$ annihilations were detected by photomultiplier tubes at both ends of the bars. Their arrival-time difference permitted the annihilation vertices to be constructed and the annihilation position to be determined with a precision of $\approx 20 \mathrm{~cm}$.

Another detector was installed to monitor extracted $\bar{p}$ beam profiles, which was a microchannel plate (MCP) with a delay line anode used as a position sensitive detector (PSD), located $1.75 \mathrm{~m}$ downstream from the center of the MRT, that is, in the middle of the beam line. An additional plastic scintillator plate near the MCP-PSD identified the $\bar{p}$ 
annihilations on the MCP-PSD by detecting passage of annihilation products such as energetic pions.

The signals from the detectors were acquired by a CAMAC system with a crate controller (TOYO CC/ NET). The crate controller had a single board computer with $320 \mathrm{MB}$ memory. The timing signals of the scintillators and the MCP-PSD were obtained by a time-to-digital converter module LeCroy 2277 and their pulse heights were determined by an analog-to-digital converter module, LeCroy $2249 \mathrm{~W}$. Because of the sufficient memory size of the single board computer, an entire data set was accumulated on that memory in order to reduce interruptions by data transfers during the cycle of MUSASHI operation, and was then transferred to another data storage computer via Ethernet.

\section{PERFORMANCE OF MUSASHI}

\section{A. Confinement of large number of $\bar{p} \mathbf{s}$}

The decelerated $\bar{p}$ beam from the RFQD passed through the Bo-PET foils. Although the foils were some 800 times thinner than those used at the entrance of the traps described in Refs. [15-17], $\bar{p}$ s traversing them suffered a finite mean energy loss (approximately $100 \mathrm{keV}$ ). Figure 12 shows the annihilation fraction in the foils as a function of the injection energy, $E_{\mathrm{RFQD}}$. The fraction monotonically decreases for higher $E_{\mathrm{RFQD}}$. The corresponding angular divergence and straggling caused only $30 \%-40 \%$ of the decelerated $\bar{p}$ s at $>110 \mathrm{keV}$ to be lost at the Bo-PET foils, while $99.9 \%$ in total were lost for the case of stopping the direct beam from the $\mathrm{AD}$ at $5.3 \mathrm{MeV}$ by relatively thick degrader foils. Figure 13 shows the longitudinal energy distribution of $111.5 \mathrm{keV} \bar{p}$ beam after passage through the foils. The $\bar{p}$ beam energy was degraded to less than $12 \mathrm{keV}$. The peak energy was around $7 \mathrm{keV}$.

For the best $\bar{p}$ trapping efficiency, the beam must be injected on-axis. Figures 14(a)-14(d) show examples of

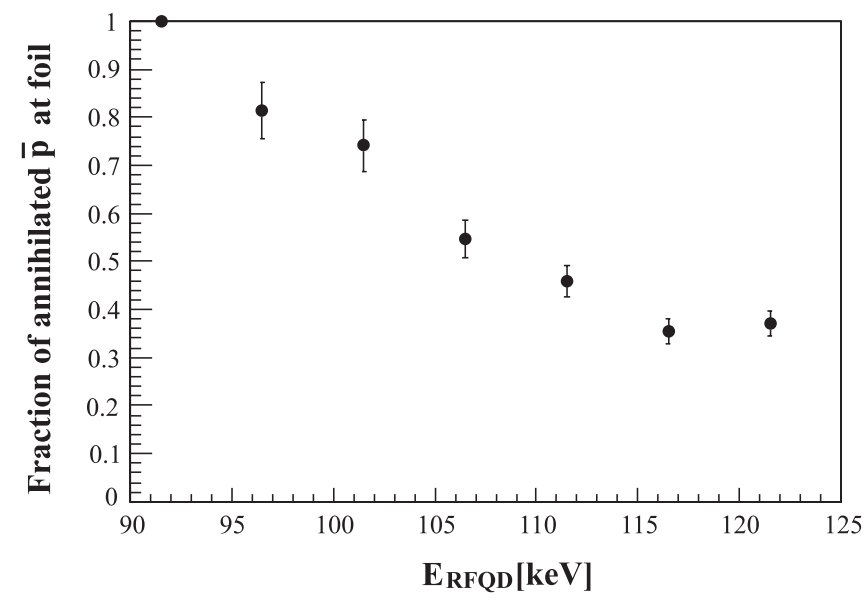

FIG. 12. Annihilated fraction of RFQD-decelerated $\bar{p}$ beam in the PET foil against their injection energy.

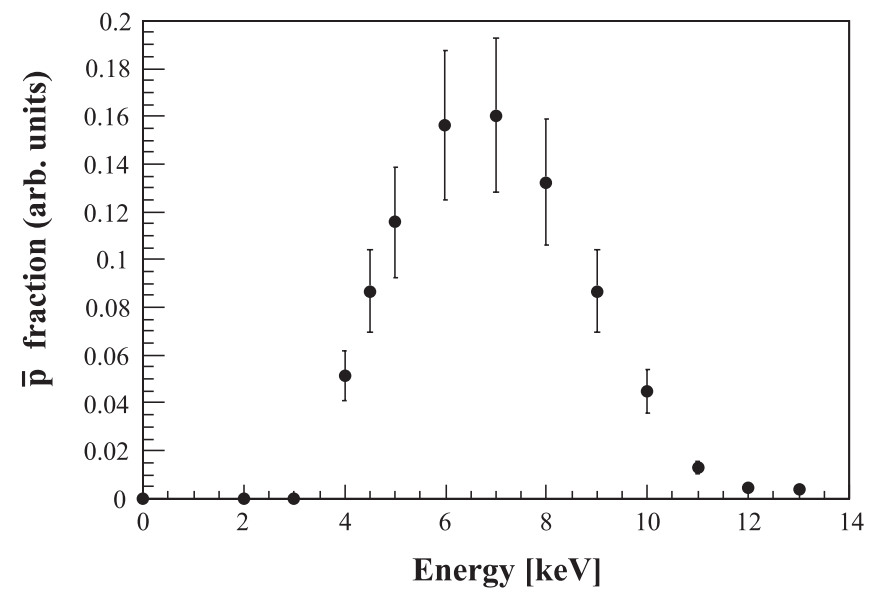

FIG. 13. The energy distribution of $111.5 \mathrm{keV} \bar{p}$ beam after passage through the foils from the RFQD.

$x$ - and $y$-projected beam profiles at the foil detector for the case of off-axis and on-axis injection. Figure 14(e) shows the integrated annihilation count when the beam was injected on an angle resulting at $5 \mathrm{~mm}$ off along the $x$ axis and $2.5 \mathrm{~mm}$ off along the $y$ axis like Figs. 14(a) and 14(b). The trapped $\bar{p}$ number, $N_{t, \text { off }}$, was only half of the initial annihilation counts $N_{i, \text { off }}$. One possible reason was its relatively larger transverse momentum due to the angle against the MRT axis. The beam was centered with its diameter of about 2-3 mm FWHM after the tuning with the LEBT elements as shown in Figs. 14(c) and 14(d). In this case, as shown in Fig. 14(f), the number of initial annihilations, $N_{i, \text { on }}$, was suppressed while the trapped $\bar{p}$ number, $N_{t, \text { on }}$ became larger than for the off-axis injection.

The $\bar{p}$ s decelerated in the foils were captured in the MRT, which stored typically $1.5 \times 10^{6}$ among the captured $\bar{p}$ s after the cooling by collisions with the $e^{-}$plasma. The introduced $\bar{p}$ beam heated up the $e^{-}$plasma before a thermal equilibrium was reestablished. The $\bar{p}$ energy reached equilibrium in the sub-eV region after 30-40 s.

Since the lifetime of the $\bar{p}$ cloud was more than a few hours, accumulation of several AD shots enabled us to store more $\bar{p} \mathrm{~s}$ [28]. Figure 15 shows the results of $7 \mathrm{AD}$ shots accumulated. The number of stored $\bar{p} \mathrm{~s}$ in the harmonic potential well was proportional to the number of $\mathrm{AD}$ shots and reached $1.05 \times 10^{7}$.

\section{B. Compression of the $\bar{p}$ cloud and extraction as ultraslow $\bar{p}$ beams}

In order to increase the number of $\bar{p}$ s extracted from the strong magnetic field of the MRT, a rotating wall technique was applied. Compression of $\bar{p} \mathrm{~s}$ after $e^{-}$removal was observed for a range of frequencies from the sideband frequency of $200 \mathrm{kHz}$ to more than $1000 \mathrm{kHz}$ without strong resonant structures [14].

Figures 16(a)-16(d) show the MCP-PSD images of extracted $\bar{p}$ beams for the application time of the rotating 
off-axis injection

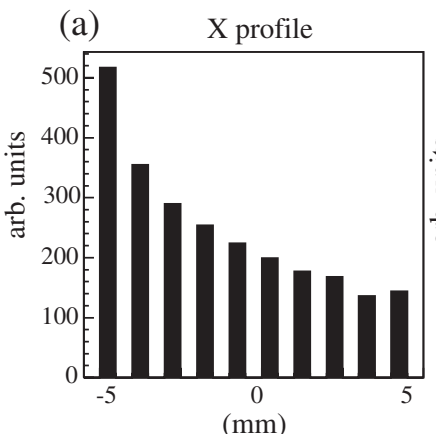

(e)
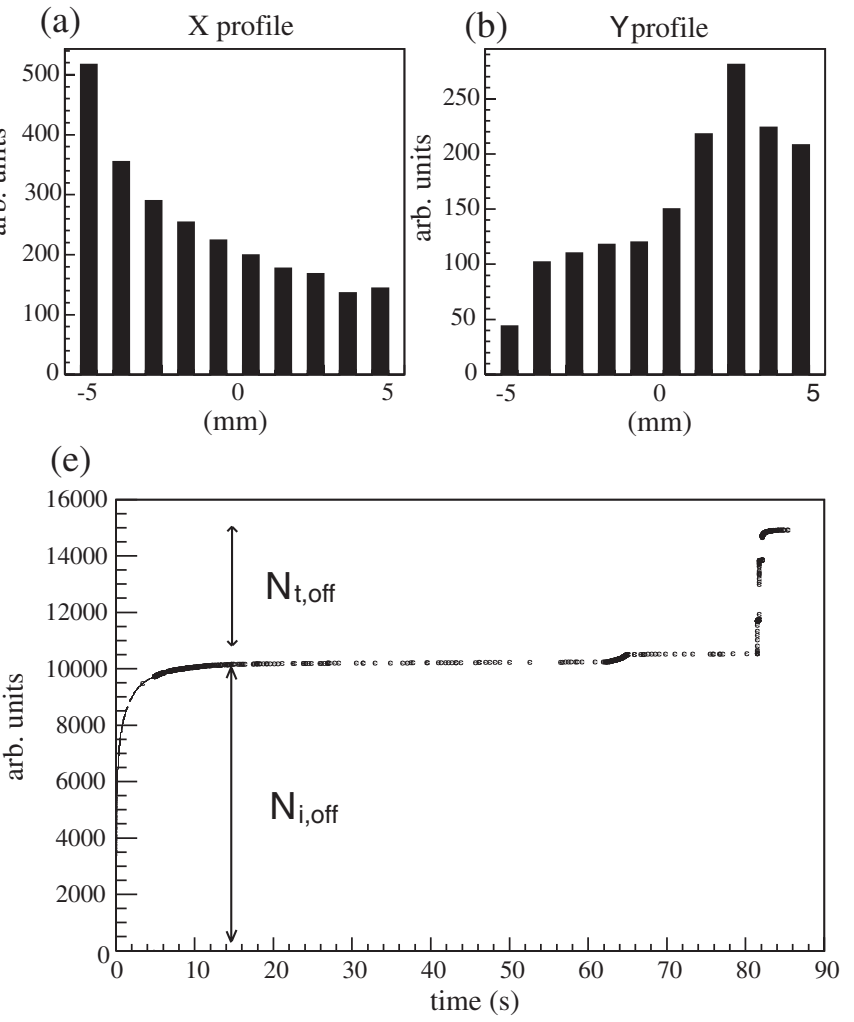

on-axis injection
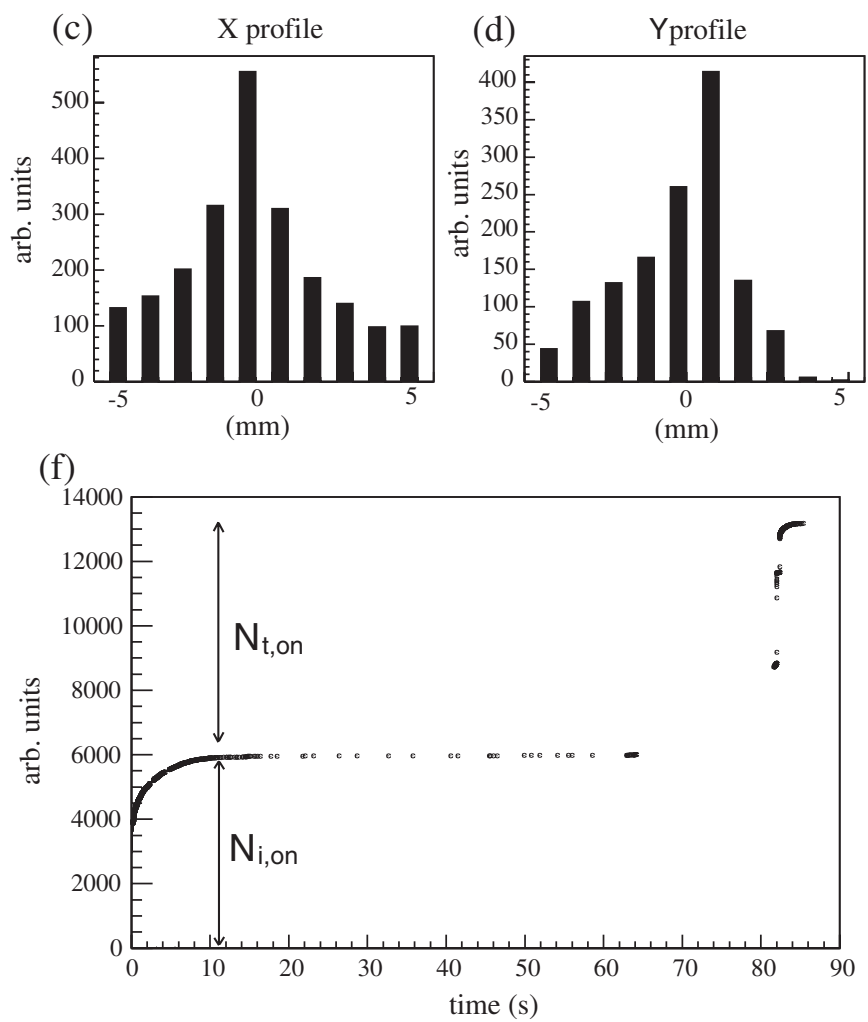

FIG. 14. (a), (b) Injected $\bar{p}$ beam $x / y$-projected profiles read by the foil detector, respectively, and (e) is the annihilation count of $\bar{p}$ for the off-axis injection case. Parts (c), (d), and (f) are in the case of on-axis injection.

wall $t_{r}=0,60,120$, and $200 \mathrm{~s}$, respectively. Without the rotating wall as shown in case (a), the image is dim with low intensity and shows a hollow depopulated region in its center. This gradually fills up as the rotating wall is applied, as seen in cases (b), (c), and (d). The cloud of $\bar{p}$ s was therefore effectively being compressed by the rotating wall. The measured extraction efficiency $\varepsilon$, defined as the ratio of the number of detected $\bar{p}$ by the MCP-PSD to the number of trapped $\bar{p}$, is shown in Fig. 16(e). The efficiency $\varepsilon$ monotonically increased from 0.08 for $t_{t}=0$ to more than 0.4 for $t_{r}>200 \mathrm{~s}$. From a comparison with observed images and a trajectory simulation assuming a $\bar{p}$ cloud being a superposition of two Gaussian distributions [14], we concluded that the cloud of $\bar{p}$ s after radial compression had a compressed thin component $(r \sim 0.25 \mathrm{~mm})$ and a halo component $(r \sim 4.0 \mathrm{~mm})$.

We also applied the rotating wall technique to a $\bar{p}$ cloud overlapping with an $e^{-}$plasma. The rotating wall tuned to $2-3 \mathrm{MHz}$ was the same as that for pure $e^{-}$plasma compression. The rotating wall amplitude having any effect on radial compression was limited to around $0.05 \mathrm{~V}_{\mathrm{pp}}$, though a rotating wall after $e^{-}$removal worked for wider range of amplitude, 0.05-0.3 $\mathrm{V}_{\mathrm{pp}}$ [14]. In addition, as was already reported in Ref. [29], the rotating wall technique with $e^{-}$plasmas worked only with a relatively long time compression, that is, the $\bar{p}$ cloud does not follow the $e^{-} \mathrm{s}$ with a quick compression.

Thus compressed, the $\bar{p}$ cloud was extracted. For collision experiments, the MUSASHI worked in the DC extraction mode by gradually decreasing the depth of the trapping

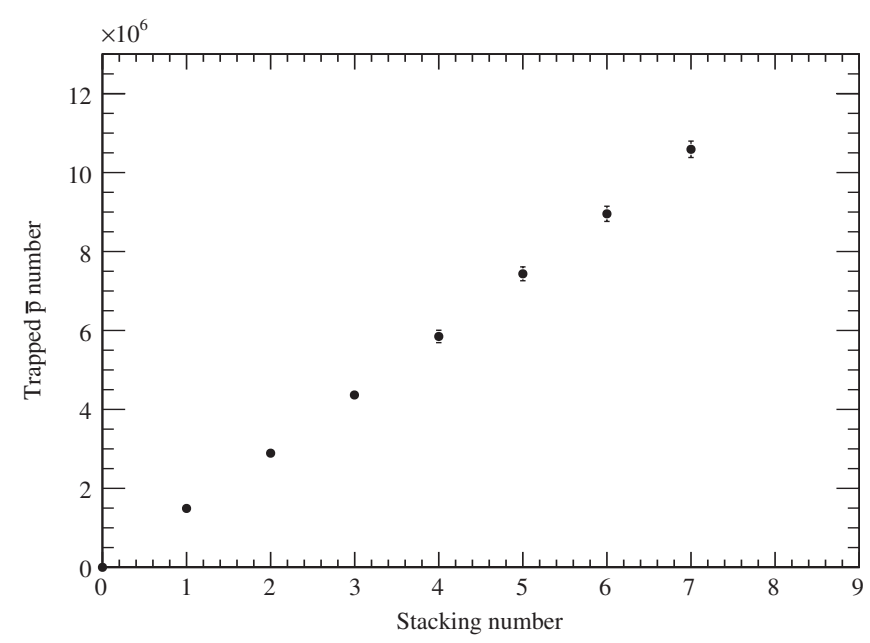

FIG. 15. Trapped $\bar{p}$ number after accumulation of several AD shots. The number of stored $\bar{p}$ was $1.05 \times 10^{7}$ after the accumulation of $7 \mathrm{AD}$ shots. 

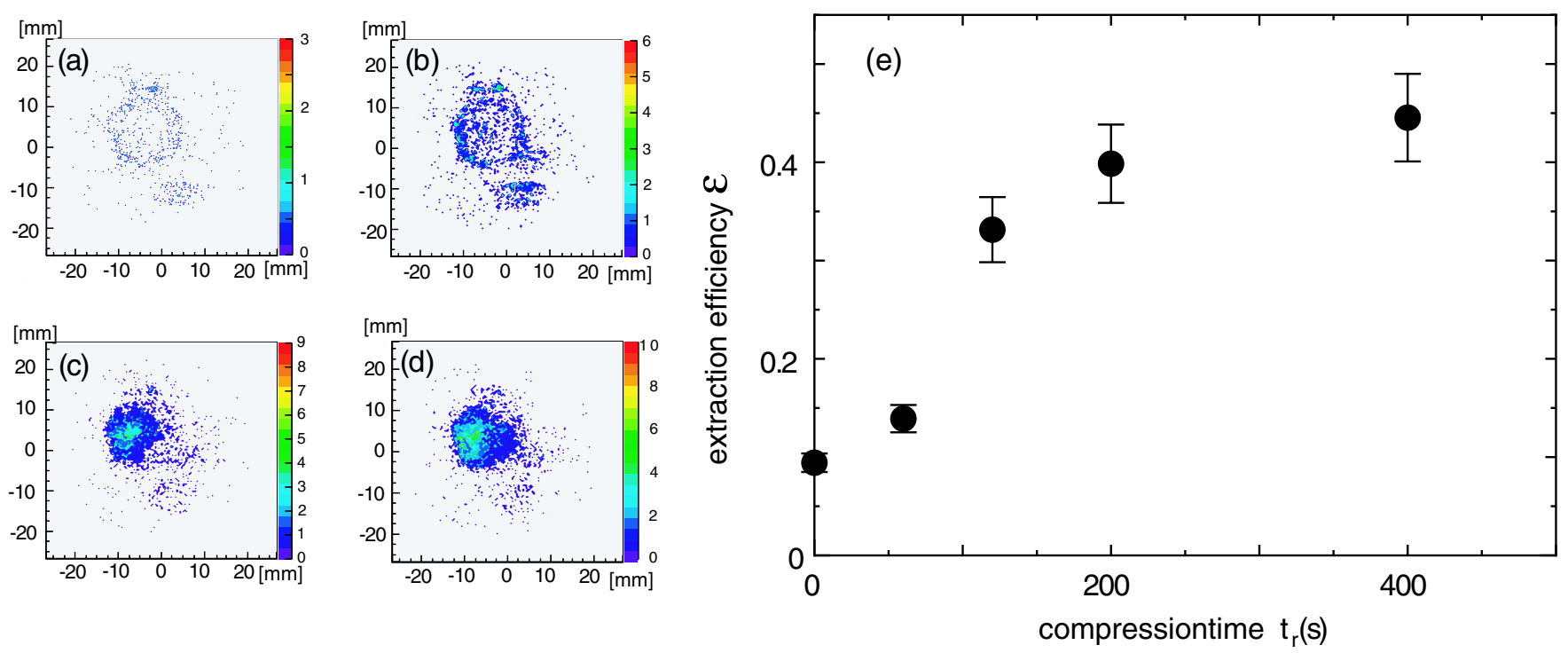

FIG. 16. (a)-(d) The PSD images of extracted $\bar{p}$ for $t_{r}=0,60,120$, and $200 \mathrm{~s}$. (e) The extraction efficiency $\varepsilon$ as a function of the compression time $t_{\mathrm{r}}$. The rotation frequency was $f=247 \mathrm{kHz}$ and the peak-to-peak amplitude was $V_{\mathrm{r}}=0.56 \mathrm{~V}$.

potential. Figure 17(a) shows a time profile of an $\bar{p}$ beam for the DC extraction. The $\bar{p}$ beam lasted up to $30 \mathrm{~s}$.

Figure 17(b) shows an energy distribution of extracted $\bar{p}$ s estimated from the potential difference between the barrier and the base of the trapping potential well. Though some hot $\bar{p}$ s remained at 3-4 V depth which was
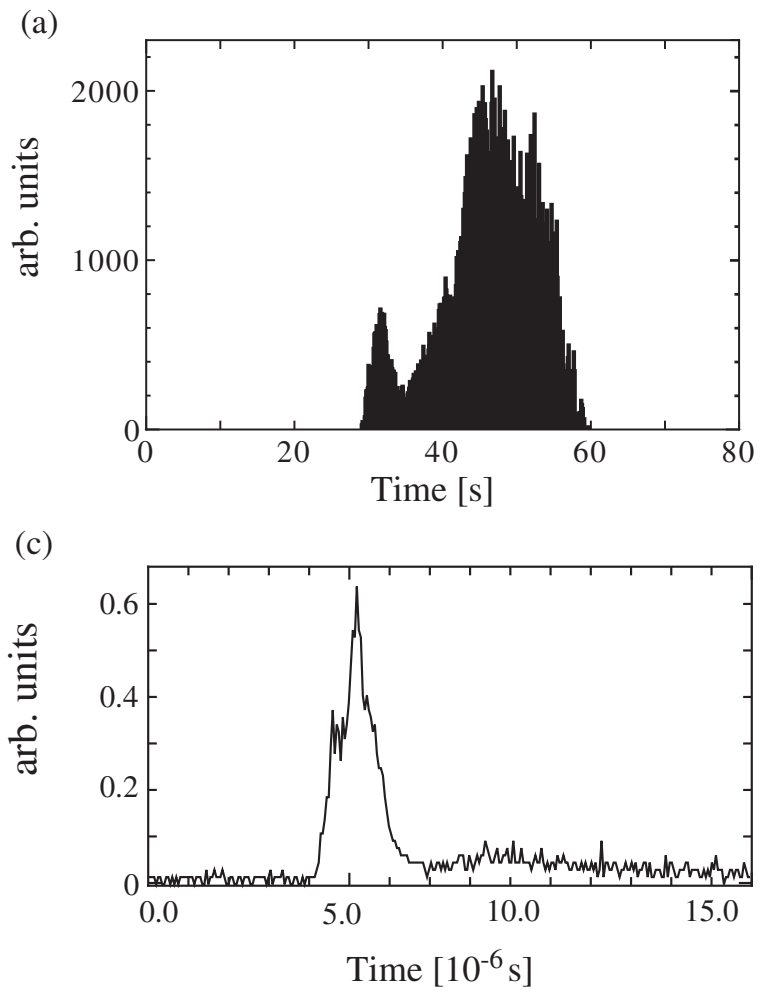

less than $10 \%$ of the total number, most of the $\bar{p}$ started to escape from the trap region when the potential depth came at around $1 \mathrm{~V}$. The total energy width of the extracted $\bar{p}$ beam would therefore be around $1 \mathrm{eV}$. It is noted that the self-field potential is $\sim 1 \mathrm{~V}$ for the compressed cloud of $\bar{p}$ for $r=0.25 \mathrm{~mm}$.

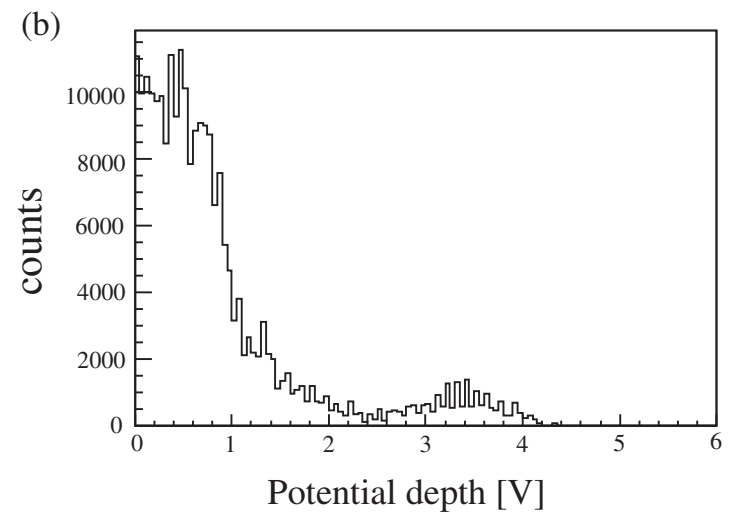

FIG. 17. (a) Typical time profile of extracted $\bar{p}$ beams for DC extraction. (b) The number of extracted $\bar{p}$ against potential depth of the MRT. (c) Typical time profile for pulsed extraction. 
The cloud of $\bar{p}$ s is also extracted as a short bunched beam by the pulse extraction mode for transportation to another trap such as the cusp trap. The radially compressed cloud was pushed into a small potential well and kicked off from that well. As shown in Fig. 17(c), the pulse length of the extracted $150 \mathrm{eV}$ beam was about $2 \mu \mathrm{s}$ at the cusp trap center $3 \mathrm{~m}$ away from the center of the MRE.

\section{SUMMARY}

The ASACUSA collaboration has developed the MUSASHI ultraslow $\bar{p}$ beam source which is based on a multiring trap consisting of multiple ring electrodes and a superconducting solenoid. A large number of $\bar{p} \mathrm{~s}, 1 \times 10^{7}$, were confined and cooled in the MUSASHI. The rotating wall technique enabled us to increase extraction efficiency. Two types of beam transport lines were developed: one was designed for gaseous targets, and the other for any experiments under ultrahigh vacuum conditions. The MUSASHI with those beam lines provided $\bar{p}$ beams of 150 or $250 \mathrm{eV}$ kinetic energy with any bunch lengths, from $2 \mu$ s to $30 \mathrm{~s}$.

\section{ACKNOWLEDGMENTS}

The authors are most grateful to the AD and RFQD staff at CERN for providing the $\bar{p}$ beam. The authors should also acknowledge ASACUSA collaboration members, in particular all former MUSASHI members, for their help. This work was supported by the Grant-in-Aid for Creative Scientific Research (No. 10P0101), the Grant-inAid for Specially Promoted Research (No. 19002004) of the Japanese Ministry of Education, Culture, Sports, Science and Technology (Monbukakagu-shō), Special Research Projects for Basic Science of RIKEN.

[1] M. Amoretti et al., Nature (London) 419, 456 (2002).

[2] G. Gabrielse et al., Phys. Rev. Lett. 89, 213401 (2002).

[3] Y. Enomoto et al., Phys. Rev. Lett. 105, 243401 (2010).

[4] R. Hughes, Hyperfine Interact. 76, 1 (1993).

[5] H. A. Torii, Y. Nagata, H. Toyoda, H. Imao, N. Kuroda, V. Varentsov, and Y. Yamazaki, Hyperfine Interact. 194, 37 (2009).

[6] H. Knudsen et al., Phys. Rev. Lett. 101, 043201 (2008).

[7] H. Knudsen et al., Phys. Rev. Lett. 105, 213201 (2010).
[8] A. Trzcińska, J. Jastrzȩbski, P. Lubiński, F. Hartmann, R. Schmidt, T. von Egidy, and B. Kłos, Phys. Rev. Lett. 87, 082501 (2001).

[9] M. Wada et al., Nucl. Instrum. Methods Phys. Res., Sect. B 204, 570 (2003).

[10] C. Surko and R. Greaves, Phys. Plasmas 11, 2333 (2004).

[11] O. Kester et al., Nucl. Instrum. Methods Phys. Res., Sect. B 204, 20 (2003).

[12] Y. Yamazaki, Nucl. Instrum. Methods Phys. Res., Sect. B 154, 174 (1999).

[13] N. Kuroda et al., Phys. Rev. Lett. 94, 023401 (2005).

[14] N. Kuroda et al., Phys. Rev. Lett. 100, 203402 (2008).

[15] G. Gabrielse, X. Fei, K. Helmerson, S. Rolston, R. Tjoelker, T. Trainor, H. Kalinowsky, J. Haas, and W. Kells, Phys. Rev. Lett. 57, 2504 (1986).

[16] G. Gabrielse, X. Fei, L. Orozco, R. Tjoelker, J. Haas, H. Kalinowsky, T. Trainor, and W. Kells, Phys. Rev. Lett. 63, 1360 (1989).

[17] M. Holzscheiter, X. Feng, T. Goldman, N. King, R. Lewis, M. Nieto, and G. Smith, Phys. Lett. A 214, 279 (1996).

[18] M. Amoretti et al., Nucl. Instrum. Methods Phys. Res., Sect. A 518, 679 (2004).

[19] A. Lombardi, W. Pirkl, and Y. Bylinsky, in Proceedings of the 19th Particle Accelerator Conference, Chicago, Illinois, 2001, edited by P. Lukas and S. Webber (IEEE, Piscataway, NJ, 2001), p. 585.

[20] A. Mohri, H. Higaki, H. Tanaka, Y. Yamazawa, M. Aoyagi, T. Yuyama, and T. Michishita, Jpn. J. Appl. Phys. 37, 664 (1998).

[21] M. Hori, Nucl. Instrum. Methods Phys. Res., Sect. A 522, 420 (2004).

[22] M. Hori, Rev. Sci. Instrum. 76, 113303 (2005).

[23] A. Mohri, Y. Kiwamoto, T. Yuyama, and T. Michishita, in 29th EPS Conference on Plasma Physics and Controlled Fusion, 2002 ECA (European Physical Society, Montreux, 2002), Vol. 26B, p. 2.033.

[24] T. Ichioka, Ph.D. thesis, University of Tokyo, 2001.

[25] X.-P. Huang, F. Anderegg, E. Hollmann, C. Driscoll, and T. O’Neil, Phys. Rev. Lett. 78, 875 (1997).

[26] H. Higaki and A. Mohri, Jpn. J. Appl. Phys. 36, 5300 (1997).

[27] K. Yoshiki Franzen et al., Rev. Sci. Instrum. 74, 3305 (2003).

[28] G. Gabrielse et al., Phys. Lett. B 548, 140 (2002).

[29] G. Andresen et al., Phys. Rev. Lett. 100, 203401 (2008). 